\title{
Effects of Endoplasmic Reticulum Stress on Pulmonary Hypertension in Rat Induced by Chronic Hypoxia and Hypercapnia
}

\author{
Congcong Zhang1,2, Jingjing Zhang1, Yuanling Wu1 ${ }^{1}$, Yongyue Dai ${ }^{1}$, Lei Ying1, Wantie Wang1* \\ ${ }^{1}$ Department of Pathophysiology, Wenzhou Medical University, Wenzhou, China \\ ${ }^{2}$ Zhejiang Pharmaceutical College, Ningbo, China \\ Email: *wwt@wmu.edu.cn
}

How to cite this paper: Zhang, C.C., Zhang, J.J., Wu, Y.L., Dai, Y.Y., Ying, L. and Wang, W.T. (2018) Effects of Endoplasmic Reticulum Stress on Pulmonary Hypertension in Rat Induced by Chronic Hypoxia and Hypercapnia. Journal of Biosciences and Medicines, 6, 53-67. https://doi.org/10.4236/jbm.2018.66004

Received: September 18, 2017

Accepted: June 19, 2018

Published: June 22, 2018

Copyright $\odot 2018$ by authors and Scientific Research Publishing Inc. This work is licensed under the Creative Commons Attribution International License (CC BY 4.0).

http://creativecommons.org/licenses/by/4.0/

\begin{abstract}
Objective: To observe the pulmonary vascular remodeling in rats with pulmonary hypertension induced by hypoxia and hypercapnia, and to explore the role of endoplasmic reticulum stress in pulmonary hypertension. Methods: 1) 40 SD rats were randomly divided into four groups: normoxic control group $(\mathrm{N})$, hypoxia hypercapnia group $(\mathrm{HH})$, endoplasmic reticulum stress (ERS) inhibitor 4-phenyl butyric acid group (4-PBA), ERS pathway agonist tunicamycin group (TM). 2) The mean pulmonary arterial pressure (mPAP) and the right ventricular hypertrophy index $(\mathrm{RV} /(\mathrm{LV}+\mathrm{S}))$ were measured in each group. 3) Identification of pulmonary artery smooth muscle cells (PASMCs) in each group by immunofluorescence $\alpha$-SMA. 4) Morphological changes of lung tissue and pulmonary artery were observed by electron microscope. 5) The apoptotic index of PASMCs in each group was detected by TUNEL. 6) Reverse transcription polymerase chain reaction (RT-PCR) and Western Blot (WB) were used to detect the expression of ERS related protein and mRNA (GRP78, CHOP, JNK, Caspase-12) in each group. Results: 1) Compared with the $\mathrm{N}$ group, the $\mathrm{mPAP}, \mathrm{RV} /(\mathrm{LV}+\mathrm{S})$ and vascular wall area $(\mathrm{WA}) /$ total area (TA) value of HH group, 4-PBA group and TM group were increased $(P<$ 0.01 ), and the vascular lumen area (LA)/TA values, PASMCs apoptosis index were significantly decreased. GRP78, CHOP, JNK, Caspase-12 expression were increased, and the differences were statistically significant. 2) Compared with the $\mathrm{HH}$ group, the mPAP, $\mathrm{RV} /(\mathrm{LV}+\mathrm{S})$ and WA/TA of 4-PBA group were decreased $(P<0.01)$; the LA/TA value and PASMCs apoptosis index were increased $(P<0.05)$; and the mRNA and protein expression of CHOP, JNK, Caspase-12 and GRP78 had a significant decrease $(P<0.05) .3)$ Compared with the $\mathrm{HH}$, the $\mathrm{mPAP}, \mathrm{RV} /(\mathrm{LV}+\mathrm{S})$ and $\mathrm{WA} / \mathrm{TA}$ of TM group were increased $(P<0.05, P<0.01)$, while LA/TA were decreased $(P<0.01)$; and
\end{abstract}


PASMCs apoptotic index was increased $(P<0.01)$. Meanwhile, the mRNA expression of Caspase-12, CHOP, JNK and GRP78 was increased to varying degrees $(P<0.05)$, and the protein expression of Caspase-12, CHOP and JNK was also increased significantly $(P<0.01)$. Conclusion: Hypoxia and hypercapnia induced pulmonary vascular remodeling may be related to the proliferation of PASMCs, and ERS related factors (JNK, Caspase12 and CHOP) are involved in the regulation of hypoxic hypercapnia.

\section{Keywords}

ERS, Pulmonary Artery Hypertension, Hypoxia Hypercapnia, Rat

\section{Introduction}

Pulmonary artery hypertension (PAH) is a kind of pulmonary vascular selective remodeling disease [1]. Pulmonary artery smooth muscle cells (PASMCS) have the characteristics of proliferation and apoptosis. Moreover, the pulmonary artery remodeling blocks the vascular lumen, finally leading to right ventricular failure and premature death. Some studies have shown that a common feature of triggering or promoting PAH processes is endoplasmic stress (ERS) [2] [3]. The endoplasmic reticulum is used to detect and handle stress, and when the stress is high or endoplasmic reticulum stress is unresolved, the endoplasmic reticulum transfers the signal to mitochondria so as to initiate programmed cells death named apoptotic [4]. Endoplasmic reticulum function is affected by various stress, including reactive oxygen species, viral infections, hypoxia, calcium homeostasis and changes in cell lipids [5] [6]. Recent studies have shown that loss of function in BMPRII and the dysfunction of key protein transport can also cause endoplasmic reticulum stress [7]. In these organelles, stress manifests as the accumulation of misfolding proteins, triggering an unfolded protein response (UPR). UPR is an important part of endoplasmic reticulum stress and mainly composed of proteinkinase RNA-like endoplasmic reticulum kinase (PERK), inositol-requiring enzyme $1 \alpha$ (IRE1 $\alpha)$ and activating transcription factor 6 (ATF6) [8]. What's more, endoplasmic reticulum stress response triggers UPR by different signal transduction pathways. Under normal stress conditions, these pathways activate several transcription factors and can protect the body by reconstructing the endoplasmic reticulum steady state. However, under severe acute or chronic stress conditions, UPR directly drives mitochondria to cause apoptosis [9], and the rapid increase in GRP78 is considered to be the most sensitive sign of endoplasmic reticulum stress [10].

PERK branches are involved in the reduction of protein synthesis, activation of cell apoptosis and autophagylase degradation [11]. In hypoxic conditions, PERK activates transcription factors ATF4 and CHOP, which induces cells apoptosis [12]. IRE1 $\alpha$ branches activate two endoplasmic reticulum stress pathways: one is regulated by the XBP-1 transcription factor, which participates in 
the expression of molecular chaperones and other endoplasmic reticulum proteins; the other is by the C-Jun amino terminal kinase (JNKs) regulation [13]. The activation of JNK plays an important role in apoptosis during chronic stress [14]. Meanwhile, the over expression of IIRE $1 \alpha$ can also directly activate Caspase-12, which induces apoptosis [15]. Therefore, it can be seen that CHOP, JNK and Caspase-12 are the three major apoptotic genes related to ERS pathway.

The previous research has shown that endoplasmic reticulum stress can be alleviated by small molecule chemical chaperones such as fatty acid derivative chaperone 4-phenylbutyric acid (4-PBA) and cholic acid derivative taurine deoxycholic acid (TUDCA). Tunicamycin (TM), a nucleoside antibiotic, and the first step in inhibiting the biosynthesis of N-linked oligosaccharides in cells [16], which leads to endoplasmic reticulum or unfolded glycoprotein, gathered in the endoplasmic reticulum, and eventually caused endoplasmic reticulum stress. In this study, 4-PBA and TM were used as inhibitors and agonists of endoplasmic reticulum stress respectively to intervene in pulmonary hypertension and explore the role of ERS pathway in hypoxia and hypercapnic induced pulmonary hypertension.

\section{Materials and Method}

\subsection{Experimental Animals}

40 healthy Sprague-Dawley (SD) male rats, weighing $200 \pm 20 \mathrm{~g}$, were obtained from the Wenzhou Medical University Animal Experimental Center, animal license: SCXK (Zhejiang) 2015-0009.

\subsection{Reagents}

4-PBA and TM (Sigma, US), Fetal Bovine Serum (Gibco, USA), Dimethyl Sulfone (DMSO, Shanghai, China), Reverse Transcription Kit (Thermo, USA), Rabbit anti-rat GRP78, JNK, p-JNK, Caspase 12 primary antibody (Abcam, UK), Mouse anti-rat CHOP primary antibody (CST company, American), TBE Buffer and RT-PCR primers (Generay Biotech, China), agarose gel (Bio-Rad, USA), skim milk powder (BD company, USA).

\subsection{Rats Model Preparation}

Forty SD rats were randomly divided into four groups $(\mathrm{n}=10)$ respectively: normoxic control group (N), hypoxia hypercapnia group (HH), ERS inhibitor 4-phenyl butyric acid group (4-PBA), ERS pathway agonist tunicamycin group (TM). The latter three groups were placed in chamber of hypoxia hypercapnia, Oxygen concentration in the tank remained at $9 \%-11 \%$, and the concentration of carbon dioxide was maintained at 5\% - 6\% (Cabin water vapor was absorbed by anhydrous $\mathrm{CaCl}_{2}$, and excess carbon dioxide was absorbed by calcium hydroxide) for 24 days. Besides, 4-PBA group was injected intraperitoneally with 4-PBA which dissolved in warm saline, the dosage was $80 \mathrm{mg} / \mathrm{kg} /$ day, and TM group was injected intraperitoneally with TM diluted with physiological saline, 
the dosage was $0.08 \mathrm{mg} / \mathrm{kg}$, twice a week. What's more, HH group intraperitoneal injection of the same amount of normal saline, and $\mathrm{N}$ group placed outside the cabin for 24 days.

\subsection{Pressure Detection}

After the rats were fixed, the pulmonary arterial mean pressure (mPAP) were measured by Powerlab bioinformatics system in vivo, then, Cut the heart and weighed the weight of the right ventricle(RV) and left ventricle plus free wall (LV $+\mathrm{S})$, then the right ventricular hypertrophy index $[\mathrm{RV} /(\mathrm{LV}+\mathrm{S})]$ were measured.

\subsection{Immunofluorescence Assay}

$\alpha$-SMA is a specific marker of smooth muscle cells, using immunofluorescence $\alpha$-SMA labeling method to identify the lungs and small arterial smooth muscle cells.

\subsection{The Observation of Transmission Electron Microscopy}

Morphological changes of lung and small arteries were observed by electron microscope.

\subsection{The Detection of TUNEL}

The apoptotic index of pulmonary artery smooth muscle cells was detected by TUNEL detection.

\subsection{RT-PCR and Western Blotting Detection}

Use reverse transcription polymerase chain reaction (RT-PCR) and Western blotting (WB) to detect the mRNA and protein expression of GRP78, CHOP, JNK and Caspase-12 in each group.

\subsection{Statistical Analysis}

SPSS19.0 software was used for statistical analysis, Metrological data were tested for normality, and data were expressed as mean \pm SD. Comparison between groups using one-way analysis of variance (one-way ANOVA). LSD method t-test was used for homogeneity of variance, and $P<0.05$ was statistically significant.

\section{Results}

\subsection{Comparison of the mPAP and RV/ $(L V+S)$ in Each Group}

Compared with $\mathrm{N}$ group, the $\mathrm{mPAP}$ and $\mathrm{RV} /(\mathrm{LV}+\mathrm{S})$ in $\mathrm{HH}$ group, 4-PBA group and TM group were significantly higher $(P<0.01)$. Compared with $\mathrm{HH}$ group, the mPAP and $\mathrm{RV} /(\mathrm{LV}+\mathrm{S})$ were lower $(P<0.01)$ in 4-PBA group, however, these indexes were increased in TM group $(P<0.05)$.

\subsection{Immunofluorescence of Pulmonary Arterioles in Each Group}

Use Rabbit anti-rat alpha-smooth muscle actin (alpha-SMA) monoclonal anti- 
body and TRITC-labeled secondary antibody. Moreover, red fluorescent markers are pulmonary artery smooth muscle cells in the figures. Compared with $\mathrm{N}$ group, the smooth muscle cells of HH group, 4-PBA group and TM group were obviously proliferated, the wall thickness was increased (vascular wallarea (WA)/total area (TA), $P<0.01$ ), and the lumen area was reduced (vascular lumen area $(\mathrm{LA}) / \mathrm{TA}, P<0.01)$. Compared with HH group, the proliferation of smooth muscle cells was inhibited by 4 -PBA group, WA/TA value was decreased, and LA/TA value was increased $(P<0.01)$. Meanwhile, the pulmonary arterial smooth muscle layer of TM group was increased, WA/TA value was increased, and LA/TA value was decreased compared with HH group $(P<0.01)$ (Figure 1, Table 1).

\subsection{Observation of Lung Tissue Transmission Electron Microscopy in Each Group}

As shown in Figure 2, pulmonary artery basement membrane epithelial cells flatted tightly adhesion in $\mathrm{N}$ group, and the microvilli emerged in the free surface of the cell, smooth muscle cells were small, and organelles were abnormal. Moreover, in the HH group, the pulmonary artery endothelial cells were damaged, Smooth muscle cells seem hypertrophy, and cytoplasm can be seen within a large number of vacuoles. Meanwhile, pulmonary artery endothelial cell were swelled and smooth muscle cells were in the contraction phenotype in 4-PBA group. Pulmonary artery endothelial cells shedding significantly, Alveolar septal vascular wall can be seen as myofibroblast proliferation in TM group (Figure 3).
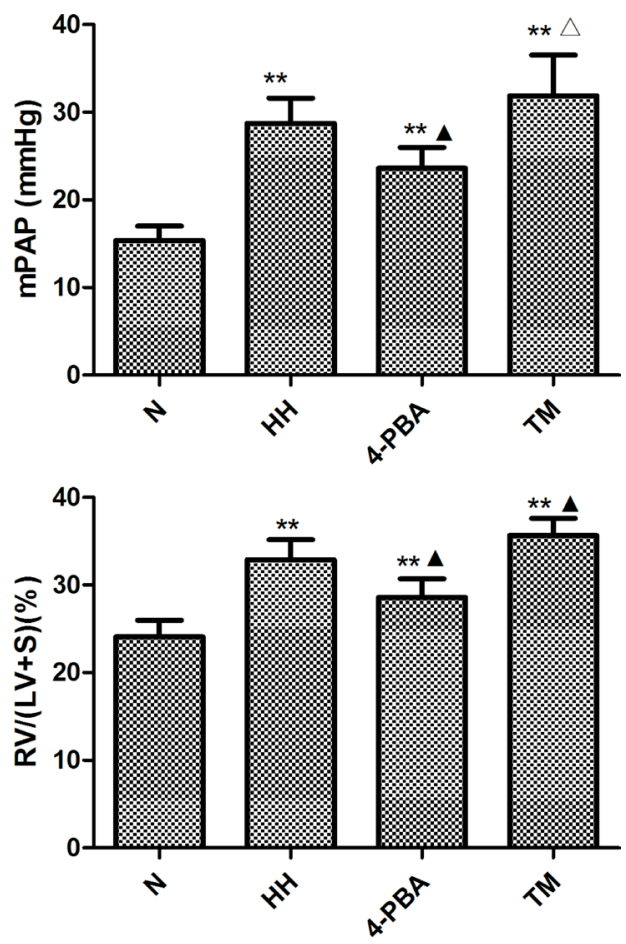

Figure 1. Expression of mPAP and RV/ $(\mathrm{LV}+\mathrm{S})$ in each group $(\bar{x} \pm \mathrm{s}, \mathrm{n}=10) .{ }^{*} P<0.05$ VS N group; ${ }^{*} P<0.01$ VS N group; $\triangle P<0.05$ VS HH group; ${ }^{\wedge} P<0.01$ VS HH group. 
Table 1. Comparison of WA/TA and LA/TA values in each group $(\bar{x} \pm \mathrm{s}, \mathrm{n}=10)$.

\begin{tabular}{|c|c|c|}
\hline Team & WA/TA (\%) & LA/TA (\%) \\
\hline $\mathrm{N}$ & $30.48 \pm 6.83$ & $65.52 \pm 6.43$ \\
\hline $\mathrm{HH}$ & $64.71 \pm 5.42^{\star \star}$ & $37.29 \pm 5.42^{\star \star}$ \\
\hline 4-PBA & $45.96 \pm 5.31^{\star \star \star}$ & $53.23 \pm 5.01^{\star \star \star}$ \\
\hline $\mathrm{TM}$ & $79.16 \pm 4.64^{* \star \Delta}$ & $23.84 \pm 3.60^{* \star \Delta}$ \\
\hline
\end{tabular}

${ }^{* *} P<0.01$ VS N group; ${ }^{\wedge} P<0.01$ VS HH group.

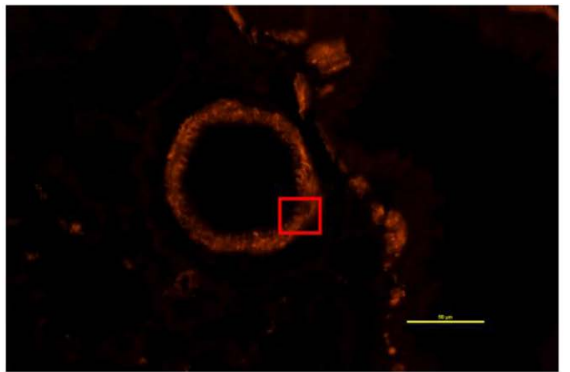

N

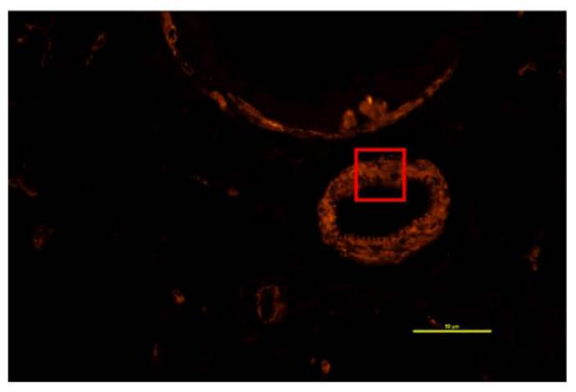

4-PBA

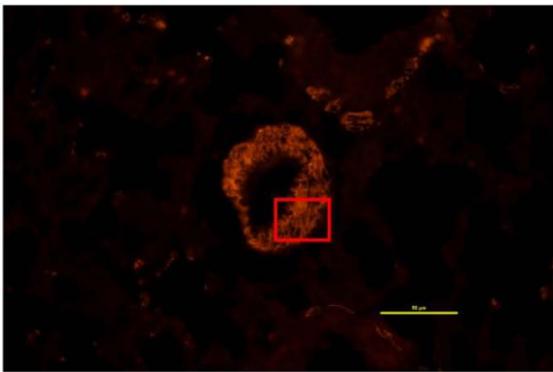

$\mathrm{HH}$

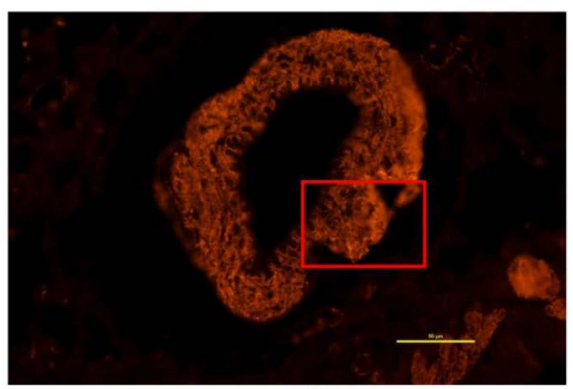

TM

Figure 2. Immunofluorescence of small pulmonary arteries in each group $(\times 400)$.

\subsection{TUNEL Identification of Pulmonary Artery Smooth Muscle Cell Apoptosis}

The apoptotic index of $\mathrm{HH}$ group, 4-PBA group and TM group were decreased compared with $\mathrm{N}$ group $(P<0.01, P<0.05)$. Moreover, the apoptotic index of 4-PBA group were increased compared with $\mathrm{HH}$ group $(P<0.05)$ and the apoptotic index of TM group was decreased $(P<0.01)$. The differences were statistically significant (Figure 4).

\subsection{Comparison of GRP78, JNK, Caspase 12 and CHOP mRNA Expression in Rats}

The expression of GRP78, JNK, Caspase12 and CHOP mRNA in HH group, 4-PBA group and TM group were all increased compared with $\mathrm{N}$ group, and the difference was statistically significant. Moreover, compared with $\mathrm{HH}$ group, the expression of JNK, Caspase12, CHOP, GRP78 mRNA in 4-PBA group was slightly lower $(P<0.05)$. Meanwhile, compared with $\mathrm{HH}$ group, the expression of JNK, Caspase12, GRP78 and CHOP mRNA in TM group was significantly up-regulated $(P<0.05, P<0.01)$ (Figures 5-8). 


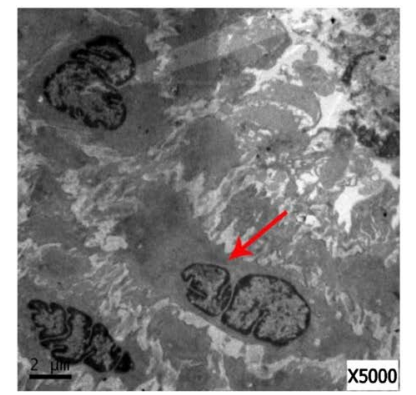

$\mathrm{N}$

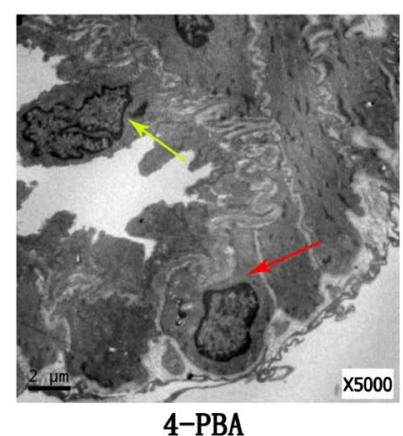

4-PBA

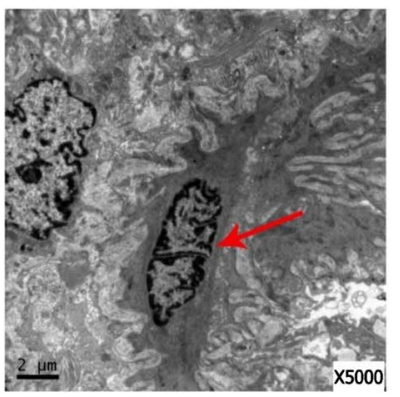

$\mathrm{HH}$

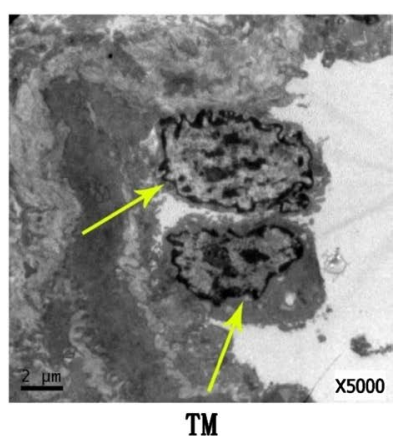

Figure 3. Transmission electron microscopic observation of lung tissue in each group $(\times 5000)$ (red arrow pointing to smooth muscle cells, yellow arrows pointing to endothelial cells).
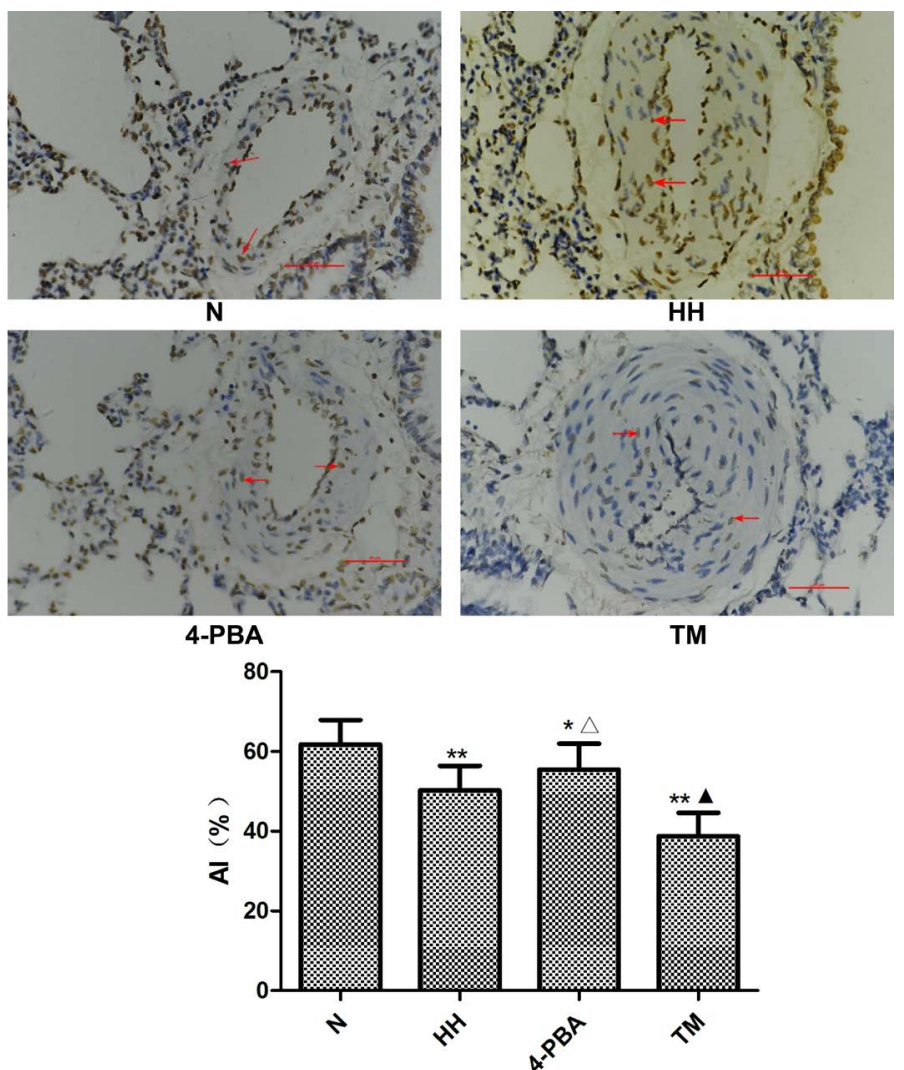

${ }^{\star} P<0.05$ VS N group; ${ }^{*} P<0.01$ VS N group; ${ }^{\triangle} P<0.05$ VS. HH group; ${ }^{\wedge} P<0.01$ VS HH group.

Figure 4. Apoptosis of pulmonary artery smooth muscle cells in each group $(\times 400)$ (red arrow pointing to positive cells). 


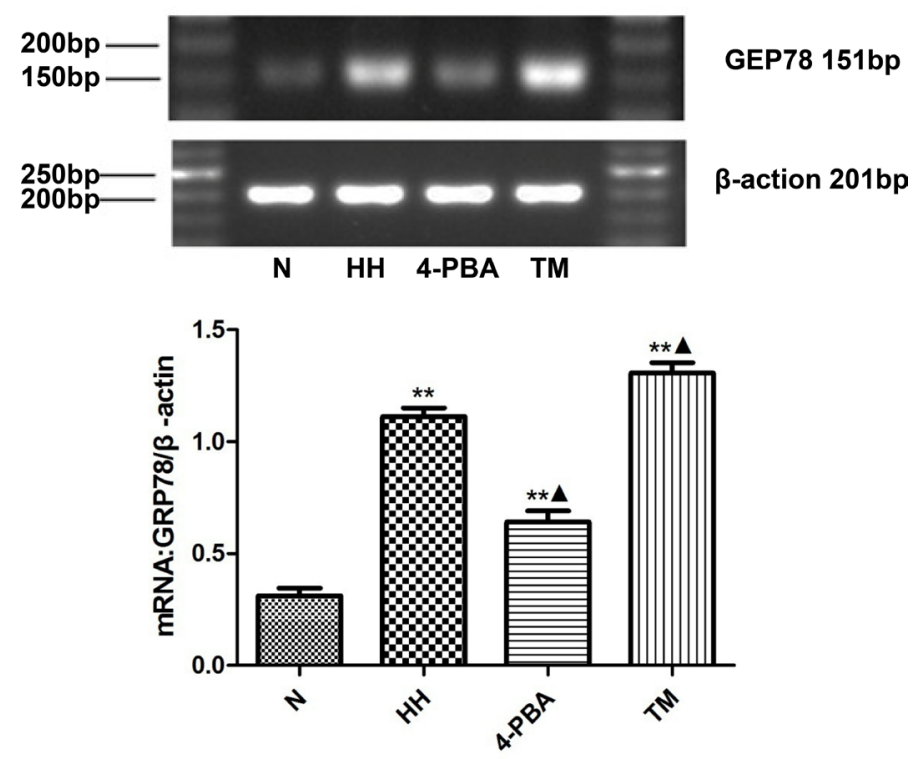

${ }^{* *} P<0.01$ VS N group; ${ }^{\mathbf{}} P<0.01 \mathrm{VSHH}$ group.

Figure 5. GRP78 mRNA expression in each group.

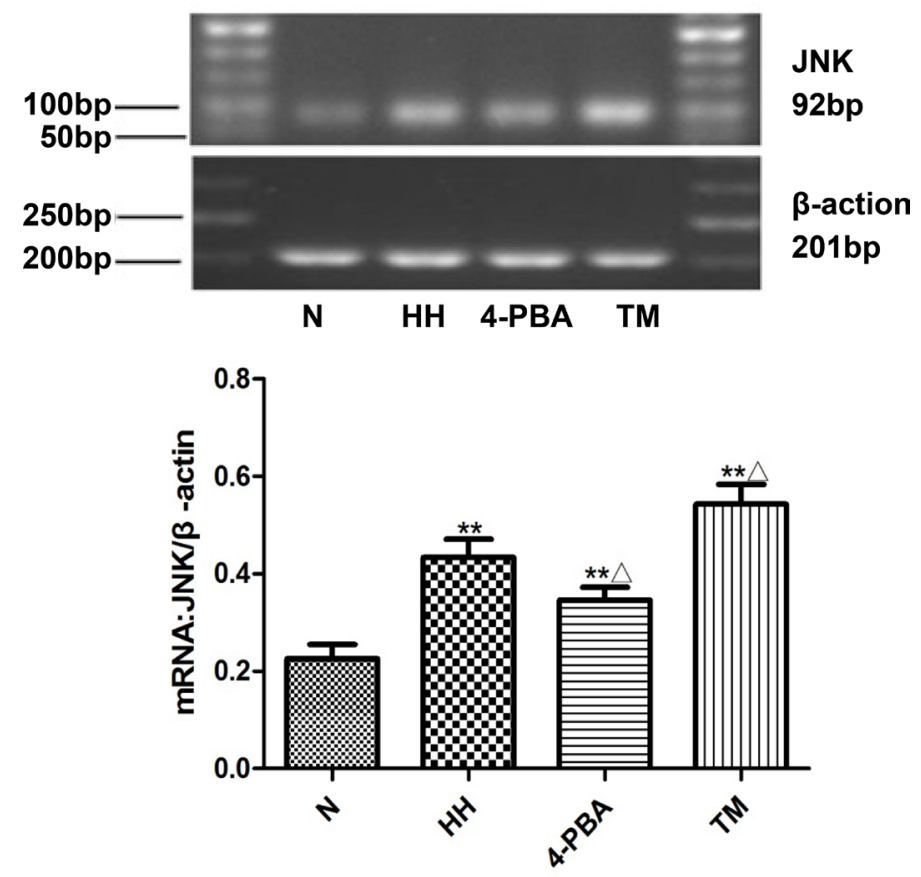

${ }^{*} P<0.01$ VS N group; $\triangle P<0.05$ VS HH group.

Figure 6. JNK mRNA expression in each group.

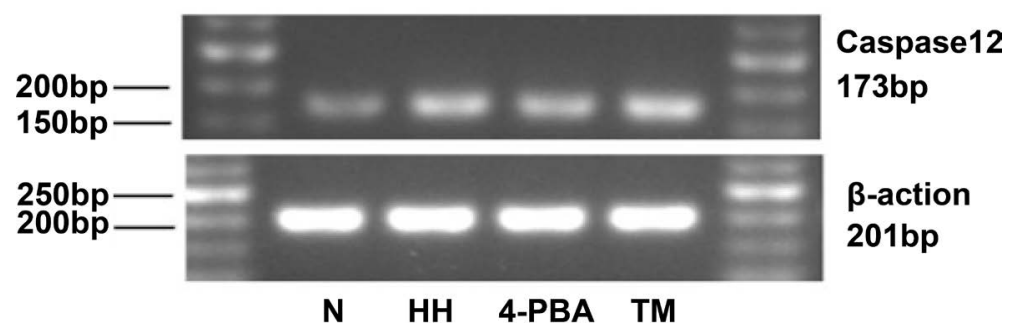




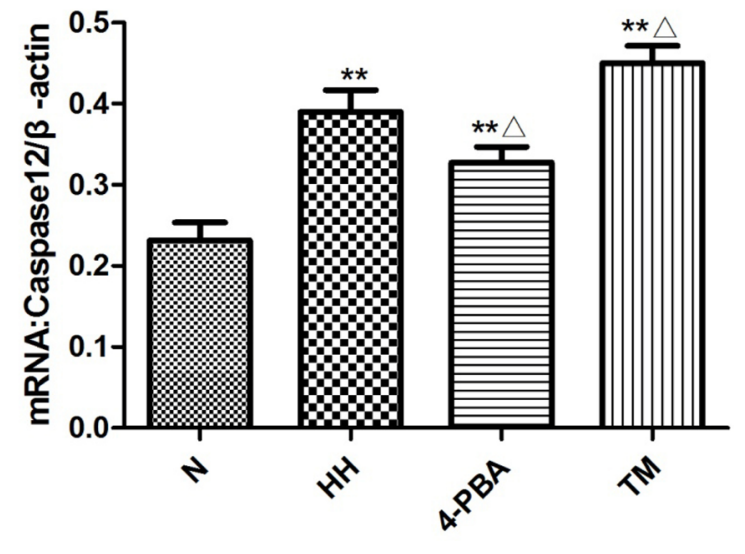

${ }^{\star *} P<0.01$ VS N group; $\triangle P<0.05$ VS HH group.

Figure 7. Caspase-12 mRNA expression in each group.
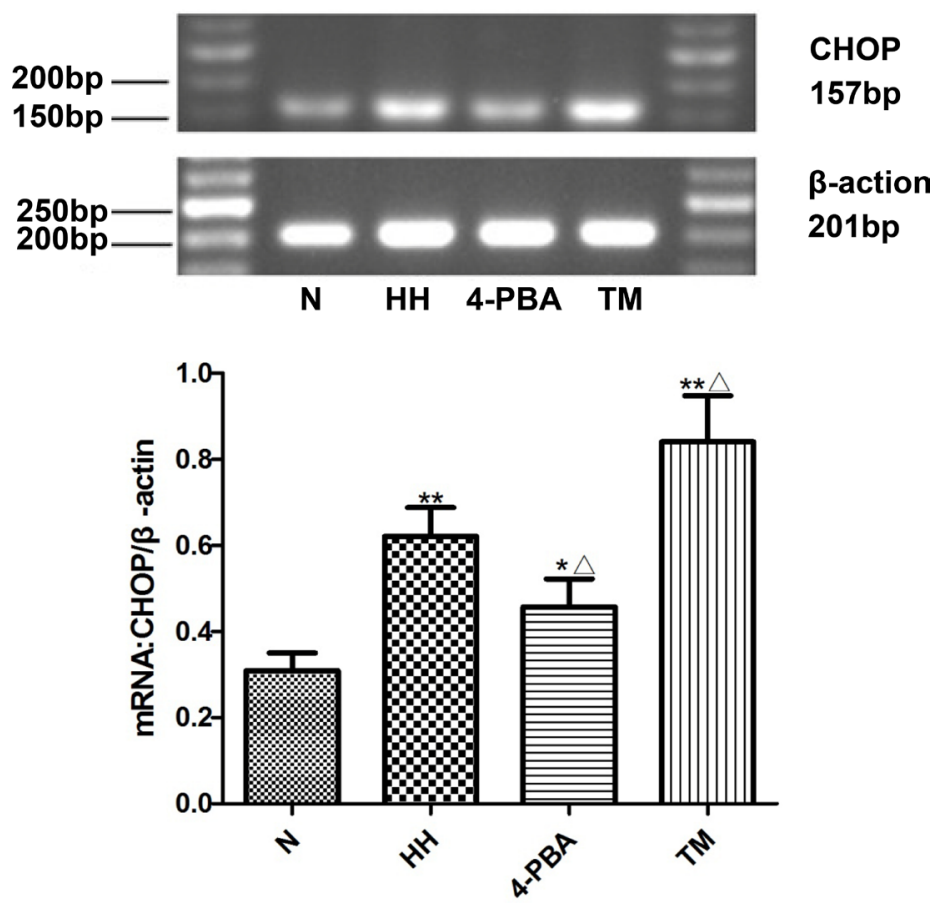

${ }^{*} P<0.01$ VS N group; $\triangle P<0.05$ VS HH group.

Figure 8. CHOP mRNA expression in each group.

\subsection{Comparison of Changes of GRP78, JNK, Caspase12 and CHOP Protein Expression in Lung Tissue of Rats}

The protein expression levels of GRP78, JNK, Caspase12 and CHOP were increased in $\mathrm{N}$ group, $\mathrm{HH}$ group, 4-PBA group and TM group compared with $\mathrm{N}$ group, and the differences were statistically significant. Moreover, compared with $\mathrm{HH}$ group, the protein expression of GRP78, JNK, Caspase12 and CHOP in 4-PBA group were decreased $(P<0.05)$, and the expression of JNK, Caspase 12 and CHOP in TM group were increased $(P<0.05, P<0.01)$, the expression of GRP78 protein was slightly up-regulated, however, the difference was not statistically significant $(P>0.05)$ (Figures 9-12). 


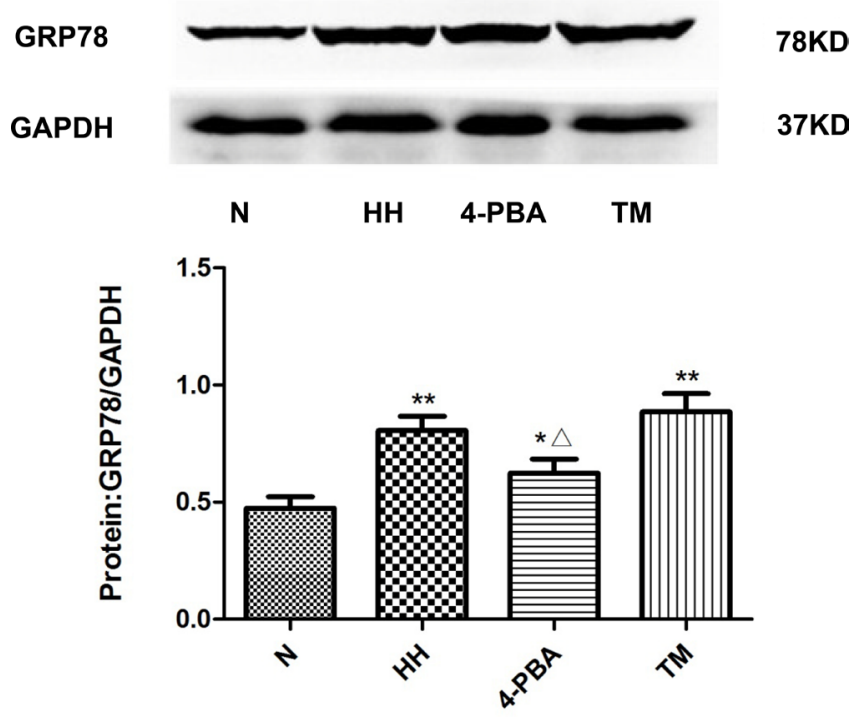

${ }^{\star} P<0.05$ VS N group; ${ }^{* *} P<0.01$ VS N group; $\triangle P<0.05$ VS HH group.

Figure 9. GRP78 protein expression in each group.
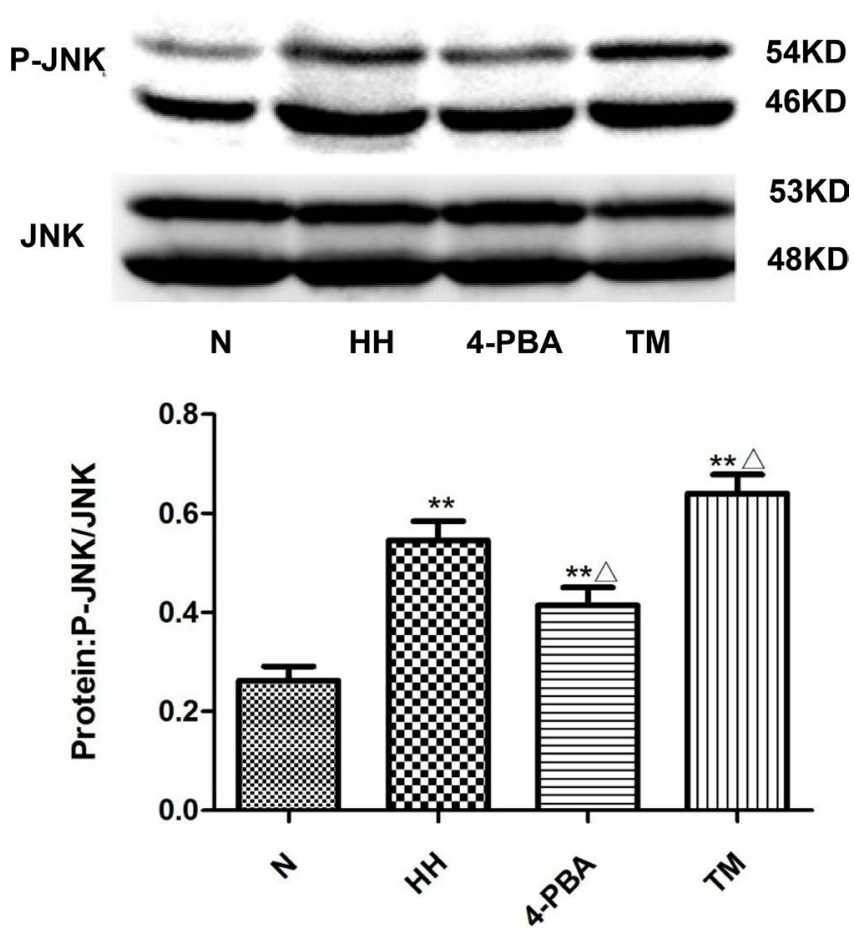

${ }^{* *} P<0.01$ VS N group; ${ }^{\triangle} P<0.05$ VS HH group

Figure 10. JNK protein expression in each group.

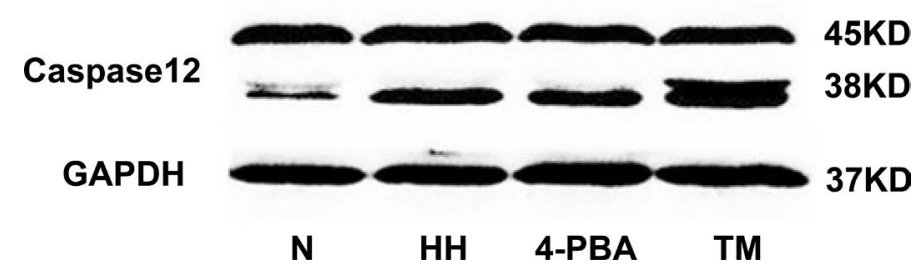




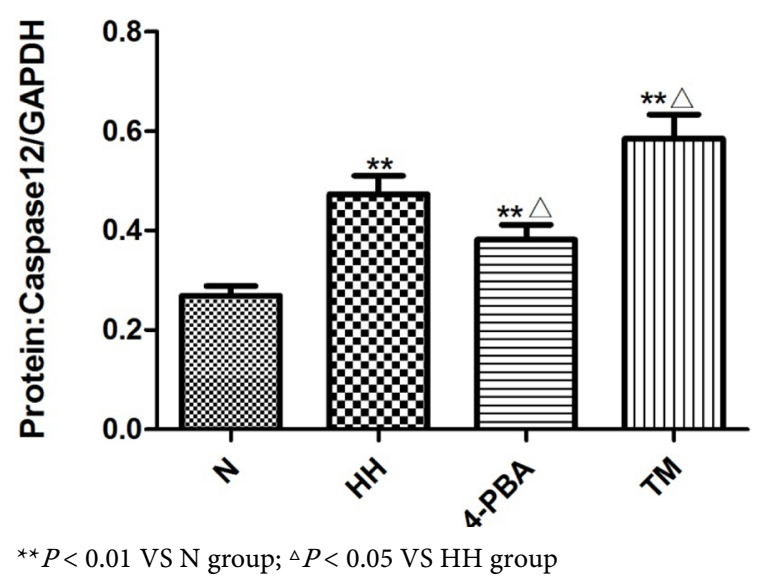

Figure 11. Caspase-12 protein expression in each group.
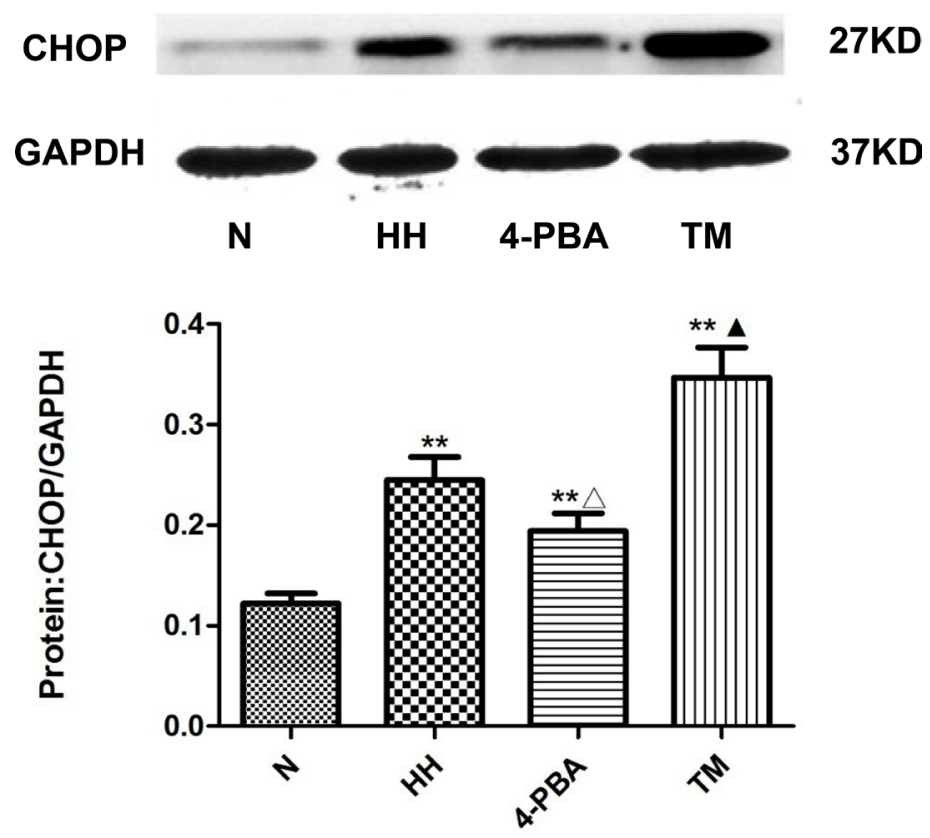

${ }^{* *} P<0.01$ VS N group; ${ }^{\circ} P<0.05$ VS HH group; ${ }^{\wedge} P<0.01$ VS HH group.

Figure 12. CHOP protein expression in each group.

\section{Discussion}

Endoplasmic reticulum is an important organelle of calcium storage, multicellular biofibroma synthesis, protein folding and secretion [17] [18]. Cell metabolism is related to endoplasmic reticulum structure, so the more exuberant metabolism, the more rich structure. Endoplasmic reticulum stress response is a mechanism for repairing false folding proteins during physiological stress, and when the pressure is irreparable, the body starts a death procedure. Moreover, chronic hypoxia-induced pulmonary hypertension model is a mature method, however, PAH patients are usually accompanied by alveolar and blood carbon dioxide partial pressure increased, therefore, in this study, we used 5\% - $6 \% \mathrm{CO}_{2}$ and $9 \%-11 \% \mathrm{O}_{2}$ mixed gas to induce rat pulmonary hypertension models. 
Although it is not clear how 4-PBA participated in cell stress response mechanisms [19], the efficacy of 4-PBA in reducing ERS in organizations has been widely demonstrated [20] [21]. So we used 4-PBA as an ERS inhibitor in this study, and from pre-experimental and some related references, the dosage of 80 $\mathrm{mg} / \mathrm{kg} /$ day was the most suitable. Moreover, N-glycosylation is a highly regulated and critical step in the process of transmembrane glycoprotein maturation [22]. TM, a nucleoside antibiotic, was a first step in inhibiting the biosynthesis of $\mathrm{N}$-oligosaccharides in cells which resulting in glycoprotein folding and causing the aggregation of the wrong or unfolded glycoprotein on the endoplasmic reticulum, and finally leaded to ER stress [23] [24]. The previous study has shown that TM enhanced the sensitivity of lung cancer cells [25]. In this study, TM was used as ERS agonist in the model rats, also from pre-experimental and some related references, the dosage of $0.08 \mathrm{mg} / \mathrm{kg}$, twice a week was better for experimental results.

In the present study, immunofluorescence, electron microscopy and TUNEL were used to observe the remodeling of pulmonary vascular remodeling. Combined with WA/TA values and LA/TA values, electron microscopic observations and apoptotic index analysis, it is suggested that hypoxia and hypercapnia-induced pulmonary vascular remodeling may be related to the proliferation of PASMCs and ERS pathway. At the same time, we also studied the regulation of ERS pathway in hypoxic hypercapnic pulmonary hypertension rats, the results showed that the expression of ERS-related protein and mRNA (GRP78, JNK, Caspase 12 and CHOP) were significantly increased in hypoxia and hypercapnia condition $(P<0.01)$. After application of ERS inhibitor 4-PBA, The expression of these proteins and mRNAs decreased respectively, and the difference was statistically significant. The protein and mRNA expression of JNK and Caspase 12 in TM group were higher than that in $\mathrm{HH}$ group $(P<0.05)$, and the protein expression of GRP78 was higher than that in HH group, but the difference was not statistically significant. What's more, in multicellular eukaryotes, endoplasmic reticulum stress is induced by three upstream signaling proteins (IRE- $1 \alpha$, PERK and ATF6), and these three pathway co-formed the UPR. Under normal circumstances, IRE- $1 \alpha$, PERK and Bip/GRP78 combined into a complex in an inactive state [26], however, when a stress response occurs, unfolded proteins or misfolding proteins accumulated in the endoplasmic reticulum, and three transmembrane proteins were activated by separation of Bip/GRP78, so the rapid upregulation of GRP78 marked the beginning of ERS [27]. Some studies have shown that endoplasmic reticulum stress-induced upregulation of GRP78 cells is limited [28], this can explain our experimental results that the GRP78 protein expression levels in TM group compared with $\mathrm{HH}$ group was no significant difference. Also, the results also showed that CHOP expression in TM group was significantly higher than that in $\mathrm{HH}$ group $(P<0.01)$. As we all know, CHOP was one of the highest inducible genes in endoplasmic reticulum stress which could promote apoptosis [29]. CHOP as a nuclear transcription factor, and un- 
der normal circumstances the protein expression was difficult to detect. In this study, we extended milk closed time, increased the anti-dilution concentration and extended the exposure time and other methods to detect it.

Studies have pointed out that reduced ERS can prevent the progress of pulmonary hypertension which mainly by inhibiting the proliferation of PASMCs and induce them apoptosis. In this study, the apoptotic index of PASMCs in $\mathrm{HH}$ group was lower than that in N group, the apoptotic index of PASMCs in 4-PBA group was higher than that in HH group, and the apoptotic index of PASMCs in TM group was higher than that in $\mathrm{HH}$ group, so the reason may be that when ERS was enhanced, the content of transmembrane protein ATF- 6 was significantly increased, and NOGO (nerve axon growth inhibitor) was released in a large amount [30]. Meanwhile, NOGO can cause mitochondrial dysfunction in PASMCs, and leaded to the transformation of glycolytic phenotype, also promoted the proliferation of PASMCs and inhibit its apoptosis, In contrast, when ERS was attenuated, it inhibited PASMCs proliferation and leaded to apoptosis [31].

\section{Summary}

Hypoxia and hypercapnia-induced pulmonary vascular remodeling may be associated with excessive proliferation and reduced apoptosis of PASMCs, and ERS-related factors (JNK, Caspase12 and CHOP) are involved in the regulation of hypoxic hypercapnic pulmonary hypertension. Therefore, we hypothesize that inhibition of ERS-related factors may be a new and effective target for the treatment of pulmonary hypertension.

\section{Acknowledgements}

This research project was supported by Wenzhou high-level talent innovation technology key projects (Grant No. 2016-07) and the Key Research Program of Traditional Chinese Medicine in Zhejiang Province (Grant No. 2013ZZ011, 2018ZZ018).

\section{References}

[1] Farha, S., Hu, B., Comhair, S., et al. (2016) Mitochondrial Haplogroups and Risk of Pulmonary Arterial Hypertension. PLoS ONE, 11, e0156042. https://doi.org/10.1371/journal.pone.0156042

[2] O’Connell, C., Montani, D., Savale, L., et al. (2015) Chronic Thromboembolic Pulmonary Hypertension. Presse Medicale, 44, e409-e416. https://doi.org/10.1016/j.lpm.2015.10.010

[3] Hao, Y., Thakkar, V., Stevens, W., et al. (2015) A Comparison of the Predictive Accuracy of Three Screening Models for Pulmonary Arterial Hypertension in Systemic Sclerosis. Arthritis Research \& Therapy, 17, 7. https://doi.org/10.1186/s13075-015-0517-5

[4] Baker, S.C., Shabir, S., Georgopoulos, N.T., et al. (2016) Ketamine-Induced Apoptosis in Normal Human Urothelial Cells: A Direct, N-Methyl-d-Aspartate Recep- 
tor-Independent Pathway Characterized by Mitochondrial Stress. American Journal of Pathology, 186, 1267-1277. https://doi.org/10.1016/j.ajpath.2015.12.014

[5] Touzet, O. and Philips, A. (2010) Resveratrol Protects against Protease Inhibitor-Induced Reactive Oxygen Species Production, Reticulum Stress and Lipid Raft Perturbation. AIDS, 24, 1437-1447.

https://doi.org/10.1097/QAD.0b013e32833a6114

[6] Inan, S. and Wei, H. (2010) The Cytoprotective Effects of Dantrolene: A Ryanodine Receptor Antagonist. Anesthesia \& Analgesia, 111, 1400-1410. https://doi.org/10.1213/ANE.0b013e3181f7181c

[7] Chida, A., Shintani, M., Matsushita, Y., et al. (2014) Mutations of NOTCH3 in Childhood Pulmonary Arterial Hypertension. Molecular Genetics \& Genomic Medicine, 2, 229-239. https://doi.org/10.1002/mgg3.58

[8] Liu, J., Xiao, M., Li, J., et al. (2017) Activation of UPR Signaling Pathway Is Associated with the Malignant Progression and Poor Prognosis in Prostate Cancer. Prostate, 77, 274-281. https://doi.org/10.1002/pros.23264

[9] Poletto, V., Dragoni, S., Lim, D., et al. (2016) Endoplasmic Reticulum Ca(2+) Handling and Apoptotic Resistance in Tumor-Derived Endothelial Colony Forming Cells. Journal of Cellular Biochemistry, 117, 2260-2271. https://doi.org/10.1002/jcb.25524

[10] Xue, Q., Li, C., Chen, J., et al. (2016) The Protective Effect of the Endoplasmic Reticulum Stress-Related Factors BiP/GRP78 and CHOP/Gadd153 on Noise-Induced Hearing Loss in Guinea Pigs. Noise Health, 18, 247-255. https://doi.org/10.4103/1463-1741.192481

[11] Tardif, N., Salles, J., Guillet, C., et al. (2014) Muscle Ectopic Fat Deposition Contributes to Anabolic Resistance in Obese Sarcopenic Old Rats through eIF2alpha Activation. Aging Cell, 13, 1001-1011. https://doi.org/10.1111/acel.12263

[12] Kim, K., Kim, Y.H., Lee, S.H., et al. (2014) Effect of Exercise Intensity on Unfolded Protein Response in Skeletal Muscle of Rat. The Korean Journal of Physiology \& Pharmacology, 18, 211-216. https://doi.org/10.4196/kjpp.2014.18.3.211

[13] Chen, H., Yang, H., Pan, L., et al. (2016) The Molecular Mechanisms of XBP-1 Gene Silencing on IRE1alpha-TRAF2-ASK1-JNK Pathways in Oral Squamous Cell Carcinoma under Endoplasmic Reticulum Stress. Biomedicine \& Pharmacotherapy, 77, 108-113. https://doi.org/10.1016/j.biopha.2015.12.010

[14] Lakshmanan, A.P., Thandavarayan, R.A., Palaniyandi, S.S., et al. (2011) Modulation of AT-1R/CHOP-JNK-Caspase12 Pathway by Olmesartan Treatment Attenuates ER Stress-Induced Renal Apoptosis in Streptozotocin-Induced Diabetic Mice. European Journal of Pharmaceutical Sciences, 44, 627-634.

https://doi.org/10.1016/j.ejps.2011.10.009

[15] Sari, F.R., Watanabe, K., Widyantoro, B., et al. (2010) Partial Inactivation of Cardiac 14-3-3 Protein in Vivo Elicits Endoplasmic Reticulum Stress (ERS) and Activates ERS-Initiated Apoptosis in ERS-Induced Mice. Cellular Physiology and Biochemistry, 26, 167-178. https://doi.org/10.1159/000320548

[16] Denzel, M.S., Storm, N.J., Gutschmidt, A., et al. (2014) Hexosamine Pathway Metabolites Enhance Protein Quality Control and Prolong Life. Cell, 156, 1167-1178. https://doi.org/10.1016/j.cell.2014.01.061

[17] De Juan-Sanz, J., Holt, G.T., Schreiter, E.R., et al. (2017) Axonal Endoplasmic Reticulum $\mathrm{Ca}^{2+}$ Content Controls Release Probability in CNS Nerve Terminals. Neuron, 93, 867-881.e6. https://doi.org/10.1016/j.neuron.2017.01.010

[18] To, M., Peterson, C.W., Roberts, M.A., et al. (2017) Lipid Disequilibrium Disrupts 
ER Proteostasis by Impairing ERAD Substrate Glycan Trimming and Dislocation. Molecular Biology of the Cell, 28, 270-284. https://doi.org/10.1091/mbc.e16-07-0483

[19] Han, J., Back, S.H., Hur, J., et al. (2013) ER-Stress-Induced Transcriptional Regulation Increases Protein Synthesis Leading to Cell Death. Nature Cell Biology, 15, 481-490. https://doi.org/10.1038/ncb2738

[20] Vega, H., Agellon, L.B. and Michalak, M. (2016) The Rise of Proteostasis Promoters. IUBMB Life, 68, 943-954. https://doi.org/10.1002/iub.1576

[21] Grall, S., Prunier-Mirebeau, D., Tamareille, S., et al. (2013) Endoplasmic Reticulum Stress Pathway Involvement in Local and Remote Myocardial Ischemic Conditioning. Shock, 39, 433-439. https://doi.org/10.1097/SHK.0b013e31828e4f80

[22] Hong, Y.P., Guo, W.Y., Wang, W.X., et al. (2016) 4-Phenylbutyric Acid Attenuates Pancreatic Beta-Cell Injury in Rats with Experimental Severe Acute Pancreatitis. International Journal of Endocrinology, 2016, Article ID: 4592346.

[23] Conrad, N., Schwager, S.L., Carmona, A.K., et al. (2016) The Effect of Structural Motifs on the Ectodomain Shedding of Human Angiotensin-Converting Enzyme. Biochemical and Biophysical Research Communications, 481, 111-116. https://doi.org/10.1016/j.bbrc.2016.10.155

[24] Beretta, G.L., Benedetti, V., Cossa, G., et al. (2010) Increased Levels and Defective Glycosylation of MRPs in Ovarian Carcinoma Cells Resistant to Oxaliplatin. Biochemical Pharmacology, 79, 1108-1117. https://doi.org/10.1016/j.bcp.2009.12.002

[25] Liu, Z., Gu, H., Gan, L., et al. (2016) Reducing Smad3/ATF4 Was Essential for Sirt1 Inhibiting ER Stress-Induced Apoptosis in Mice Brown Adipose Tissue. Oncotarget, 8, 9267-9279.

[26] Lopez-Sambrooks, C., Shrimal, S., Khodier, C., et al. (2016) Oligosaccharyltransferase Inhibition Induces Senescence in RTK-Driven Tumor Cells. Nature Chemical Biology, 12, 1023-1030. https://doi.org/10.1038/nchembio.2194

[27] Ling, Y.H., Li, T., Perez-Soler, R., et al. (2009) Activation of ER Stress and Inhibition of EGFR N-Glycosylation by Tunicamycin Enhances Susceptibility of Human Non-Small Cell Lung Cancer Cells to Erlotinib. Cancer Chemotherapy and Pharmacology, 64, 539-548. https://doi.org/10.1007/s00280-008-0902-8

[28] Han, X., Zhang, X., Li, H., et al. (2015) Tunicamycin Enhances the Antitumor Activity of Trastuzumab on Breast Cancer in Vitro and in Vivo. Oncotarget, 6, 38912-38925. https://doi.org/10.18632/oncotarget.5334

[29] Kim, H., Baek, C.H., Lee, R.B., et al. (2017) Anti-Fibrotic Effect of Losartan, an Angiotensin II Receptor Blocker, Is Mediated through Inhibition of ER Stress via Up-Regulation of SIRT1, Followed by Induction of HO-1 and Thioredoxin. International Journal of Molecular Sciences, 18, 305. https://doi.org/10.3390/ijms18020305

[30] Suyama, K., Watanabe, M., Sakabe, K., et al. (2011) Overexpression of GRP78 Protects Glial Cells from Endoplasmic Reticulum Stress. Neuroscience Letters, 504, 271-276. https://doi.org/10.1016/j.neulet.2011.09.045

[31] Pinto, B.A., Melo, T.M., Flister, K.F., et al. (2016) Early and Sustained Exposure to High-Sucrose Diet Triggers Hippocampal ER Stress in Young Rats. Metabolic Brain Disease, 31, 917-927. https://doi.org/10.1007/s11011-016-9830-1 